\title{
A life threatening complication of anti reflux surgery: acute gastric volvulus
}

\author{
Mustafa özsoy ${ }^{1}$, Zehra özsoy ${ }^{2}$, Suleyman Sahin ${ }^{1}$, Yuksel Arıkan ${ }^{3}$ \\ ${ }^{1}$ Department of General Surgery, Parkhayat Hospitals, Afyon, Turkey \\ ${ }^{2}$ State Hospital, Afyon, Turkey \\ ${ }^{3}$ Faculty of Medicine, Afyon Kocatepe University, Afyon, Turkey
}

Key words: Gastric volvulus; Nissen fundoplication; antireflux surgery

\section{Introduction}

Gastric volvulus is divided into two as primary and secondary according to the aetiology. In primary gastric volvulus, the pathology is the relaxation of the support ligaments of stomach. Secondary gastric volvulus develops due to predisposing factors such as hiatus hernia, traumatic diaphragmatic rupture, diaphragm eventration, intrinsic and extrinsic pathologies of stomach, and abdominal adhesions [1].

\section{Case presentation}

A 27-year-old male patient was admitted with a sudden onset of chest pain, nausea, and inability to vomit. His medical history revealed that he underwent a laparoscopic Nissen fundoplication operation six months ago. Laboratory results were as follows: aspartate aminotransferase: $45 \mathrm{U} / \mathrm{L}$, alanine aminotransferase: $100 \mathrm{U} / \mathrm{L}$, total bilirubin: $0,9 \mathrm{mg} / \mathrm{dL}$, amylase: $200 \mathrm{U} / \mathrm{L}$, creatinine: $1,4 \mathrm{mg} / \mathrm{dL}$, leukocyte count: 12 $10^{3} / \mu \mathrm{L}$. Initially, unstable angina was suspected; however, electrocardiography and troponin I $(0.3 \mathrm{mg} / \mathrm{L})$ value of the patient were found to be normal. On the chest X-ray, air-fluid level was detected behind the heart, and it was observed in the computed tomography that the stomach was herniated into the thoracic cavity with organoaxial rotation (Figure 1).
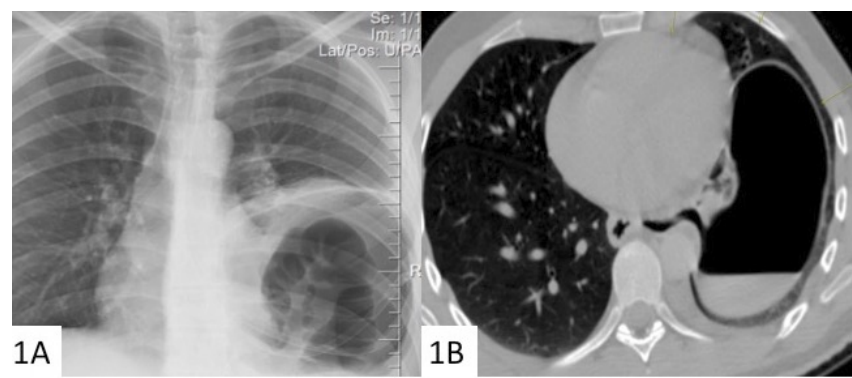

Figure 1A. Chest X-ray showing air fluid level 1B. computed tomography showing acute gastric volvulus

Correspondence: Mustafa özsoy

E-mail: dr.mustafaozsoy@gmail.com

Received: 03-03-2018 Accepted: 25-03-2018

(iD) http://orcid.org/0000-0003-0098-3855

DOI: http://doi.org/10.4038/sljs.v36i1.8481
The patient was diagnosed with acute gastric volvulus, since the nasogastric catheter was unable to be inserted, and it was decided to perform endoscopic detorsion. Gastric ischaemia was not observed during the endoscopy, although detorsion failed and the patient underwent emergency surgery. In the laparoscopic exploration, it was observed that along with the stomach, colon was also herniated through the diaphragmatic defect into the thorax. Due to the failure of detorsion of stomach and colon and the difficulty of dissection, it was decided to convert to conventional surgery. The stomach and colon were pulled into the abdomen. After the stomach was detorsioned, the hernia sac was resected. The diaphragmatic defect was closed with primary suturing and repaired with the aid of a mesh. To avoid gastric detorsion, gastropexy was performed fixing the greater curvature of the stomach to the abdominal wall with $2 / 0$ silk (Figure 2). The mesocolon was also fixed to the retrocolic area using primary suturing. On the fourth postoperative day, the patient was uneventfully discharged.

\section{Discussion}

Acute gastric volvulus is characterized by Borchardt's triad; severe epigastric abdominal pain, distention and unproductive vomiting [2]. The clinical presentation of chronic gastric volvulus is rather obscure. It is encountered with various findings such as atypical chest pain, anaemia (often related to Cameron's ulcer), weight loss, dysphagia and reflux. If the diagnosis of gastric volvulus is delayed, fatal outcomes may occur such as ulceration, haemorrhage, ischaemia and up to full-thickness necrosis [3].

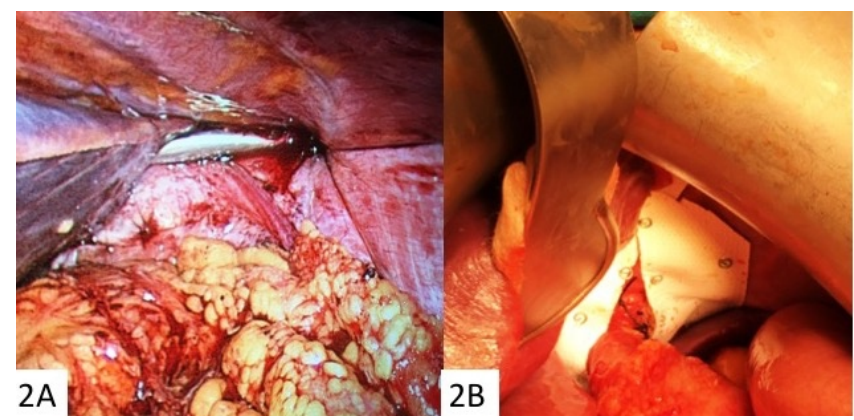

Figure 2 A. Laparoscopic exploration showing thoracic herniation of the stomach and colon $\mathbf{2 B}$. Reconstruction of large diaphragmatic defect with a mesh is seen. 
The incidence of strangulation in gastric volvulus cases ranges from $5 \%$ to $28 \%$ [4]. Acute gastric volvulus development after laparoscopic Nissen fundoplication is an extremely rare clinical entity. If there is a delay in diagnosis, strangulation is likely to be detected. The relaxation of gastro splenic, gastro colic and gastro hepatic ligaments which hold the stomach in place and postoperative adhesions are responsible in the pathogenesis [5]. Gastric volvulus is often in the organo-axial type and the stomach takes the shape of hourglass [6]. Diagnosis is made with radiological examinations in accordance with clinical suspicion. In the early period, the increased serum amylase value from laboratory results is particularly indicative of ischaemia. Computed tomography is the gold standard radiological examination [7]. Treatment steps of gastric volvulus include correction of volvulus and prevention of recurrence. Llaneza et al. [8] described that detorsion could be achieved by nasogastric decompression in volvulus cases. However, nasogastric decompression is useful in relieving acute clinical picture rather than a definitive treatment option. Endoscopic evaluation should be performed in these patients without losing time. Thus, by determining the grade of ischaemia, loss of time is prevented. Tsang and Walker have described that endoscopic treatment is possible in acute and chronic gastric volvulus cases. The endoscope is brought to the J-shape manoeuvre in the fundus, pushed forward to the antrum and duodenum clockwise, which is called alpha-loop manoeuvre [9]. Surgical treatment is performed by laparoscopic or conventional methods. The main goals of surgical procedure are correction of volvulus, excision of hernia sac, repair of hiatus hernia and anterior gastropexy [10].

\section{Conclusion}

Acute gastric volvulus development after laparoscopic Nissen fundoplication is an extremely rare clinical entity. If the diagnosis of gastric volvulus is delayed, fatal outcomes may occur. Surgical treatment should be performed when acute gastric volvulus is detected in patients who have had previous upper abdominal surgery.
All authors disclose no conflict of interest. The study was conducted in accordance with the ethical standards of the relevant institutional or national ethics committee and the Helsinki Declaration of 1975, as revised in 2000 .

\section{References}

1. Sevcik WE, Steiner IP. Acute gastric volvulus: case report and review of the literature. CJEM 1999 Oct;1(3):200-3. PMID:17659139 https://doi.org/10.1017/S1481803500004206

2. Borchardt M. Zun pathologie and therapy des magnevolvulus. Arch Klin Chir. 1904;74:243-8

3. Luke T. Channer, MD, Gregory T. Squires, MD, Phillip D. Price, MD. Laparoscopic Repair of Gastric Volvulus. JSLS 2000;4(3): 225-30 PMCID: PMC3113174

4. Savolainen H, Lehtimäki T, Rantanen T. Gastric volvulus after laparoscopic Nissen fundoplication and hiatal closure performed due to giant hiatal hernia: A case report. J Case Rep Images Surg 2015;1:13-16. doi:10.5348/Z12-2015-4-CR-4

5. Dalgaard JB. Volvulus of the stomach. Acta Clin Scand. 1952;103(2):131. PMID: 14943396

6. Baty V, Rocca P, Fontaumard E. Acute gastric volvulus related to adhesions after laparoscopic fundoplication. Surg Endosc 2002 Mar;16(3):538doi: 10.1007/s00464-001-4226-6 https://doi.org/10.1007/s00464-001-4226-6

7. Williams L, Lansdown MR, Larvin M, Ward DC. Gastric volvulus: a rare cause of hyperamylasaemia. Br J Clin Pract 1990;44(12):708-9. PMID:1714754

8. Llaneza PP, Salt WB. Gastric volvulus. More common than previously thought. Postgrad Med. 1986;80(5):279-88. PMID:3763530 https://doi.org/10.1080/00325481.1986.11699576

9. Tsang TK, Walker R. Endoscopic reduction of gastric volvulus: the alpha-loop maneuver. Gastro Endosc. 1995;42(3):244- 248. PMID:7498690 https://doi.org/10.1016/S0016-5107(95)70099-4

10. Naim HJ, Smith R, Gorecki PJ. Emergent laparoscopic reduction of acute gastric volvulus with anterior gastropexy. Surg Laparosc Endosc Percutan Tech. 2003;13(6):389-91. https://doi.org/10.1097/00129689-200312000-00009

\section{Learning Points:}

- Acute gastric volvulus is an extremely rare but known clinical entity following laparoscopic Nissen fundoplication.

- Computed tomography is the gold standard radiological examination.

- In patients who have had previous upper abdominal surgery, surgical treatment should be performed 